\title{
History of Migraine and Volume of Brain Infarcts: The Italian Project on Stroke at Young Age (IPSYS)
}

\author{
Valeria De Giuli, ${ }^{\mathrm{a},}{ }^{*}$ Michele Besana, ${ }^{\mathrm{b},{ }^{*}}$ Mario Grassi, ${ }^{\mathrm{c}}$ Marialuisa Zedde, ${ }^{\mathrm{d}}$ Andrea Zini, ${ }^{\mathrm{e}}$ Corrado Lodigiani, ${ }^{\mathrm{f}}$ \\ Simona Marcheselli, ${ }^{\mathrm{g}}$ Anna Cavallini, ${ }^{\mathrm{h}}$ Giuseppe Micieli, ${ }^{\mathrm{i}}$ Maurizia Rasura, ${ }^{\mathrm{j}}$ Maria Luisa DeLodovici, ${ }^{\mathrm{k}}$ \\ Giampaolo Tomelleri, ${ }^{1}$ Nicoletta Checcarelli, ${ }^{\mathrm{m}}$ Alberto Chiti, ${ }^{\mathrm{n}}$ Elisa Giorli, ${ }^{\mathrm{o}}$ Massimo Del Sette, ${ }^{\mathrm{p}}$ \\ Lucia Tancredi, ${ }^{\mathrm{q}}$ Antonella Toriello, ${ }^{\mathrm{r}}$ Massimiliano Braga, ${ }^{\mathrm{s}}$ Andrea Morotti, ${ }^{\mathrm{h}}$ Loris Poli, ${ }^{\mathrm{a}}$ Filomena Caria, ${ }^{\mathrm{a}}$ \\ Massimo Gamba, ${ }^{\mathrm{t}}$ Rosalba Patella, ${ }^{\mathrm{j}}$ Alessandra Spalloni, ${ }^{\mathrm{j}}$ Anna Maria Simone, ${ }^{\mathrm{e}}$ Rosario Pascarella, ${ }^{\mathrm{d}}$ \\ Sandro Beretta, ${ }^{\mathrm{s}}$ Enrico Fainardi, ${ }^{\text {, }}$ Alessandro Padovani, ${ }^{\mathrm{a}}$ Roberto Gasparotti, ${ }^{\mathrm{b}}$ Alessandro Pezzini, ${ }^{\mathrm{a}}$ \\ on behalf of the Italian Project on Stroke in Young Adults (IPSYS) Investigators
}

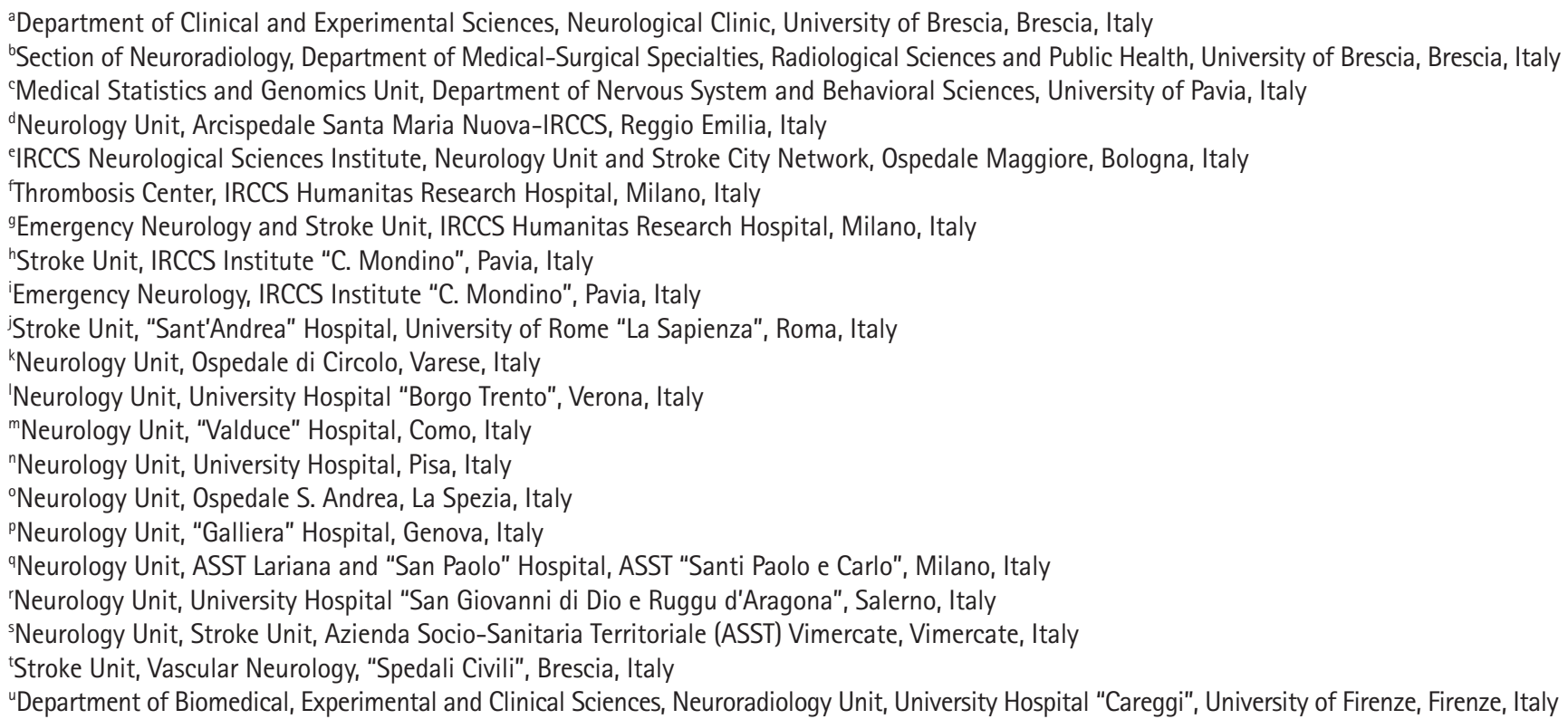

Background and Purpose Migraine has been shown to increase cerebral excitability, promote rapid infarct expansion into tissue with perfusion deficits, and result in larger infarcts in animal models of focal cerebral ischemia. Whether these effects occur in humans has never been properly investigated.

Methods In a series of consecutive patients with acute ischemic stroke, enrolled in the setting of the Italian Project on Stroke at Young Age, we assessed acute as well as chronic infarct volumes by volumetric magnetic resonance imaging, and compared these among different subgroups identified by migraine status.

Results A cohort of 591 patients (male, 53.8\%; mean age, 37.5 \pm 6.4 years) qualified for the analysis. Migraineurs had larger acute infarcts than non-migraineurs (median, $5.9 \mathrm{~cm}^{3}$ [interquartile range (IOR), 1.4 to 15.5$]$ vs. $2.6 \mathrm{~cm}^{3}$ [IOR, 0.8 to 10.1$\left.], P<0.001\right)$, and the largest volumes were observed in
Correspondence: Alessandro Pezzini Department of Clinical and Experimental Sciences, Neurological Clinic, University of Brescia, P.le Spedali Civili 1, 25123 Brescia, Italy

Tel: +39-30-3384086

Fax: $+39-30-3384086$

E-mail: alessandro.pezzini@unibs.it

Received: February 6, 2019

Revised: June 19, 2019

Accepted: July 17, 2019

*These authors contributed equally to the manuscript as first author. 
patients with migraine with aura (median, $9.0 \mathrm{~cm}^{3}$ [IQR, 3.4 to 16.6]). In a linear regression model, migraine was an independent predictor of increased log (acute infarct volumes) (median ratio [MR], $1.64 ; 95 \%$ confidence interval $[\mathrm{Cl}], 1.22$ to 2.20$)$, an effect that was more prominent for migraine with aura (MR, 2.92; 95\% Cl, 1.88 to 4.54).

Conclusions These findings reinforce the experimental observation of larger acute cerebral infarcts in migraineurs, extend animal data to human disease, and support the hypothesis of increased vulnerability to ischemic brain injury in people suffering migraine.

Keywords Brain ischemia; Cortical spreading depression; Migraine disorders; Risk factors; Stroke

\section{Introduction}

Migraine is a chronic-intermittent primary headache disorder that affects roughly $15 \%$ of the Western population. ${ }^{1}$ Although migraine, in particular migraine with aura, has been shown to be associated to ischemic stroke, ${ }^{2}$ the exact mechanisms underlying this relation remain unclear.

Potential biologic processes include endothelial dysfunction, hypercoagulability, platelet aggregation, vasospasm, shared cardiovascular risk factors and genetic risk, paradoxical embolism, and use of nonsteroidal anti-inflammatory drugs. ${ }^{3-8}$

Recent experimental evidence in mice has suggested that cerebral hyperexcitability and enhanced susceptibility to spreading depolarization, the electrophysiologic event underlying migraine, may be one additional mechanism involved in the migraine-stroke association. ${ }^{9,10}$ The increased cerebral excitability of migraineurs may facilitate infarction if and when the tissue becomes ischemic, by predisposing to frequent ischemic depolarizations. According to this pathogenic hypothesis, it might be that elevated cerebral blood flow threshold for viability, a sign of increased vulnerability to ischemia, may promote rapid infarct expansion into tissue with milder perfusion deficits, diminish salvageable tissue at risk, and result in larger infarcts in susceptible migraineurs. Currently, a paucity of data is available to substantiate this experimental model in clinical setting, ${ }^{11,12}$ and, in particular, whether patients with personal history of migraine actually develop larger infarct volumes has not been properly investigated so far. To explore this hypothesis, in the present study we, therefore, sought to determine whether acute infarct volume, as well as the volume of chronic cerebral infarcts, assessed by volumetric magnetic resonance imaging (MRI) analysis, are associated with migraine and its subtypes in a large, prospective, hospital-based cohort of young patients with acute ischemic stroke.

\section{Methods}

\section{Study design and study population}

The Italian Project on Stroke at Young Age (IPSYS) is a countrywide network of neurological centers with special interest in cerebral ischemia at young age across Italy, aimed at recruiting white patients with first-ever acute stroke who fulfill the following criteria: (1) age 18 to 45 years, (2) computed tomography (CT)- or MRI-proven cerebral infarction, in the setting of a hospital-based, multicentre, observational study ${ }^{13}$ (Appendix 1). The study was approved by the local Ethics Committee. Written informed consent was obtained for all participants (or next of kin). Centers were included in the IPSYS network provided that the recruitment process of stroke cases takes place prospectively. Stroke was defined as a sudden loss of global or focal cerebral function that persisted for $>24$ hours with a probable vascular cause. ${ }^{14}$ Ischemic stroke due to sinus venous thrombosis, vasospasm after subarachnoid hemorrhage, cardiac surgery, occurring as an immediate consequence of trauma, and iatrogenic strokes were excluded. The recruitment period for the present study was January 2006 through December 2015.

\section{Clinical and laboratory investigations}

All patients underwent an etiologic workup including complete blood cell count, biochemical profile, urinalysis, 12-lead electrocardiogram, chest roentgenography, Doppler ultrasonography with frequency spectral analysis and B-mode echotomography of the cervical arteries, transcranial Doppler ultrasonography, and CT and/or magnetic resonance angiography to investigate extracranial and intracranial vessels. Coagulation testing included prothrombin and activated partial thromboplastin times, circulating anti-phospholipid antibodies, fibrinogen, protein $C$, protein $S$, activated protein $C$ resistance, antithrombin III, genotyping to detect factor $\mathrm{V}$ Leiden and the G20210A mutation in the prothrombin gene. Transthoracic and/or transesophageal echocardiography were performed to rule out cardiac sources of emboli. Based on the results of such 
investigations, patients were classified according to a classification based on the Trial of Org 10172 in Acute Stroke Treatment (TOAST) criteria, accommodated and validated for the cause of stroke in the young ${ }^{15}$ and divided into five etiologic categories: (1) atherosclerotic vasculopathy; (2) non-atherosclerotic vasculopathy; (3) small-vessel disease; (4) cardioembolism; and (5) other: cerebral infarction that did not meet the criteria for one of the categories outlined. ${ }^{16}$

\section{Potential confounders}

Hypertension was defined as systolic blood pressure $\geq 140 \mathrm{~mm}$ $\mathrm{Hg}$ and diastolic pressure $\geq 90 \mathrm{~mm} \mathrm{Hg}$ in two separate measurements after the acute phase or use of antihypertensive drugs before recruitment. Diabetes mellitus as history of diabetes mellitus, use of hypoglycemic agent or insulin, or fasting glucose $\geq 7.0 \mathrm{mmol} / \mathrm{L}$. Smoking history was defined as "current smoking," including former smokers who had quit smoking for 6 months before the index event, versus "ever smoking" or "never smoking." Hypercholesterolemia as cholesterol serum levels $\geq 5.7 \mathrm{mmol} / \mathrm{L}$ or use of cholesterol-lowering drugs. Current use of oral contraceptives included current user as well as former users who had quit taking these medications for 1 month before the index event. Family history of stroke was defined when stroke was recorded in first-degree relatives by interviewing probands or family members. We also collected information on heavy alcohol consumption (weekly consumption $>14$ drinks for men and $>7$ drinks for women), personal history of coronary artery disease (history of physician diagnosed angina or myocardial infarction), and atrial fibrillation (medical history or electrocardiographic findings at admission).

\section{MRI protocol}

Brain MRI scans were executed on 1.0- and 1.5-tesla scanners. Image analysis was applied to the MRI closest to the onset of clinical symptoms. The MRI protocols included turbo spin echo (TSE) and fluid-attenuated inversion recovery (FLAIR) T2weighted images and diffusion-weighted images acquired on axial plane and, where available, gradient echo (GE) T2*weighted images acquired on axial plane and three-dimensional time-of-flight (3D TOF)-magnetic resonance angiography images. For each image series, 20 slices covering the entire brain (matrix $240-256 \times 256-320$; field of view 22 to $24 \mathrm{~cm}$; thickness $5 \mathrm{~mm}$; interslice gap $1.5 \mathrm{~mm}$ ) were obtained. Time repetition/time of echo/time of inversion/flip angle values were optimized on each scanner for the best available image contrast and resulted comparable across the centers.

All MRI available in electronic format were collected, securely stored, and analysed centrally (Brescia University Hospital). An acute brain infarct was defined as a non-mass parenchymal defect, hyperintense on $\mathrm{T} 2$, with a vascular distribution, with elevated signal in high b-value diffusion-weighted MRI (DWI) images and low signal in apparent diffusion coefficient (ADC) maps. A chronic brain infarct was identified as non-mass lesion, isointense to cerebral-spinal fluid in all sequences, with low signal in high b-value DWI and an elevated signal in ADC maps.

\section{Image assessment}

Magnetic resonance studies were evaluated by a neuroradiologist (M.B.) and a neurologist trained in neuroimaging (V.D.G.) who were blinded to the diagnosis and clinical data. For each patient, DWI confirmation of acute cerebral infarction was needed.

\section{Volumetric calculation of infarcted areas}

Axial T2 fluid-FLAIR images were converted from Digital Imaging and COmmunications in Medicine (DICOM) to analyze format for computer-assisted calculation of the infarct volume, using ANALYZE 11.0 (Mayo Clinic, Rochester, MN, USA). The Intensity Loaded Volume-Region of Interest function was used to optimize intensity settings, thereby enhancing contrast between gray and white matter, infarcted tissue and normal brain and assist in boundary definition. A region-of-interest map of brain infarcts was created by signal intensity thresholding on axial TSE/FLAIR images, followed by manual semiautomatic editing (Region of Interest module) and automatic calculation of the lesion volume. Number, location, and volume of acute, and chronic infarcts were recorded.

\section{Migraine assessment}

Personal history of headache was assessed in all participants by study physicians during a face-to-face interview with patients and/or family members in both acute phase and followup evaluations. The diagnosis of migraine, as well as its subtypes migraine without aura and migraine with aura was made according to the diagnostic criteria of the Headache Classification Committee of the International Headache Society. ${ }^{17}$

\section{Statistical analyses}

Infarct volumes were log-transformed for all analyses in this study. All variables were reported as either a mean value ( \pm standard deviation), a median value with an interquartile range (IQR) or a proportion/percentage of total. For descriptive analyses, we compared patients characteristics by migraine subgroups (migraine vs. non-migraine; migraine without aura vs. migraine with aura vs. non-migraine) using the chi-square test, Median-test, and Anova F-test, as appropriate. Inter-rater reliability in MRI measurements was assessed by having the 
two examiners categorize the same set of images in a subgroup of 30 patients and was calculated using interclass correlation (ICC) for single measures of acute volumes. The same approach was used to evaluate the reliability of volume calculation on FLAIR images versus DWI images. Using a linear regression model of the log (acute infarct volume) values we assessed the change in log-volumes of acute infarct volume as well as chronic infarct volume in patients with migraine (and its subtypes migraine without aura and migraine with aura) and migraine-free patients. Regression analyses were adjusted for covariates that reached a $P<0.05$ in univariate analysis. Because of their potential influence on final infarct volume we also planned to include the following variables in the model, regardless of their distribution in the two subgroups of patients defined by migraine status: (1) time from stroke onset to MRI; (2) presumed etiology of brain ischemia (categorized according to the TOAST criteria); (3) large vessel occlusion (occlusion vs. non-occlusion); (4) acute reperfusion therapy (intravenous tissue plasminogen activator and/or mechanical thrombectomy vs. no reperfusion therapy); (5) arterial recanalization (complete recanalization [defined as a Thrombolysis in Cerebral Infarction score of Illb or higher at the end of the endovascular procedure $\left.{ }^{18}\right]$ vs. other). To explore potential effect measure modification, we also performed analyses stratified by sex. Results are given as exp (beta)=median ratios (MRs) with 95\% confidence intervals (Cl). $P \leq 0.05$ on 2-sided test was considered significant. Data were analyzed using the SPSS version 21.0 software package (IBM Co., Armonk, NY, USA).

\section{Results}

Fourteen of the 22 centers included in the IPSYS network participated to the present imaging study. The remaining eight centers were unable to obtain endorsement of the MRI analysis by the local health authority. In all, 600 patients qualified for the study. Nine of these were excluded due to presence of contraindications for MRI scan (e.g., pregnancy, claustrophobia, cardiac electronic devices; $n=3$ ), because they died before brain MRI was performed $(n=2)$, or because magnetic resonance images were not suitable for volumetric measurement due to poor quality $(n=4)$. Hence, data from 591 patients (male, 53.8\%; mean age, $37.5 \pm 6.4$ years) were entered into the analysis. Of these, 160 (27.0\%) had a personal history of migraine (migraine without aura/migraine with aura, 103/57). Migraineurs were similar to non-migraine patients with regard to demographic characteristics and risk factors, although females were more prevalent in the former subgroup and they also reported to assume oral contraceptives at a higher percentage. Also, the prevalence of heavy alcohol consumers among migraineurs was lower compared with that among non-migraineurs. The etiologic categories "cardiac embolism" and "other or undetermined etiology" were the most represented among patients suffering migraine (Table 1).

Interobserver agreement was good for acute infarct volumes (ICC coefficient, $0.89 ; 95 \% \mathrm{Cl}, 0.78$ to 0.95 ). Similarly, there was a good agreement for volume measurements on FLAIR images and DWI images (ICC coefficient, $0.83 ; 95 \% \mathrm{Cl}, 0.65$ to 0.92 ). In univariate analysis, patients with personal history of migraine had larger acute infarct lesions than patients with no history of migraine (median, $\exp [8.69] / 10^{3}=5.9 \mathrm{~cm}^{3}[I \mathrm{IQR}, 1.4$ to 15.5$] \mathrm{vs.}$ $\exp [7.87] / 10^{3}=2.6 \mathrm{~cm}^{3}[$ [IQR, 0.8 to 10.1$\left.] ; P<0.001\right)$. In particular, the largest volumes were observed in the subgroup of patients with migraine with aura (median, $\exp [9.11] / 10^{3}=9.0 \mathrm{~cm}^{3}[$ [QR, 3.4 to 16.6$]$ vs. $\exp [8.2] / 10^{3}=3.6 \mathrm{~cm}^{3}[$ [IQR, 1.0 to 14.4$]$ in pa-

Table 1. Demographic and clinical characteristics of study participants by migraine status

\begin{tabular}{|c|c|c|c|}
\hline Characteristic & Migraine $(n=160)$ & $\begin{array}{c}\text { Non-migraine } \\
(n=431)\end{array}$ & $P$ \\
\hline Age (yr) & $36.7 \pm 6.2$ & $37.1 \pm 6.5$ & 0.443 \\
\hline Male sex & $69(43.1)$ & $249(57.8)$ & 0.002 \\
\hline Hypertension & $28(17.5)$ & $97(22.5)$ & 0.185 \\
\hline Diabetes & $5(3.1)$ & $19(4.4)$ & 0.482 \\
\hline Hypercholesterolemia & $50(31.3)$ & $107(24.8)$ & 0.116 \\
\hline Current smoking & $54(33.8)$ & $174(40.8)$ & 0.142 \\
\hline Oral contraceptives* & $42(46.2)$ & $60(33.0)$ & 0.046 \\
\hline Family history of stroke & 34 (21.3) & $86(20.0)$ & 0.728 \\
\hline Atrial fibrillation & $2(1.3)$ & $2(0.5)$ & 0.297 \\
\hline $\begin{array}{l}\text { Cardiac interatrial right-to- } \\
\text { left shunt }\end{array}$ & $90(56.3)$ & $116(26.9)$ & $<0.001$ \\
\hline Heavy alcohol consumption & $15(9.4)$ & 73 (16.9) & 0.022 \\
\hline Cause of stroke & & & 0.369 \\
\hline Large-vessel disease & $13(8.1)$ & $45(10.4)$ & \\
\hline $\begin{array}{l}\text { Non-atherosclerotic vas- } \\
\text { culopathies }\end{array}$ & $32(20.0)$ & $60(13.9)$ & \\
\hline Small-vessel disease & $14(8.8)$ & 49 (11.4) & \\
\hline Cardiac embolism & $50(31.3)$ & $132(30.6)$ & \\
\hline $\begin{array}{l}\text { Other or undetermined } \\
\text { etiology }\end{array}$ & $51(31.9)$ & $145(33.6)$ & \\
\hline Large artery occlusion & $12(7.5)$ & $35(8.1)$ & 0.866 \\
\hline $\begin{array}{l}\text { IV thrombolysis/endovascu- } \\
\text { lar treatment }\end{array}$ & $17(10.6)$ & $34(7.9)$ & 0.323 \\
\hline Vessel recanalization & $8(5.0)$ & $27(6.3)$ & 0.696 \\
\hline $\begin{array}{l}\text { Time from stroke onset to } \\
\text { MRI (hr) }\end{array}$ & $32.5(24.0-45.5)$ & $36.0(24.0-48.0)$ & 0.167 \\
\hline
\end{tabular}

Values are presented as mean \pm standard deviation, number (\%), or median (interquartile range).

IV, intravenous; MRI, magnetic resonance imaging.

*In females. 
tients with migraine without aura; $P<0.001$ ) (Figure 1).

These results were apparently not influenced by specific migraine characteristics (i.e., migraine activity and pain severity), though this comparison was possible only in a small subgroup of patients (Supplementary Table 1). In the linear regression model of log(acute infarct volume) values including age, alcohol consumption, as well as all the variables with a potential

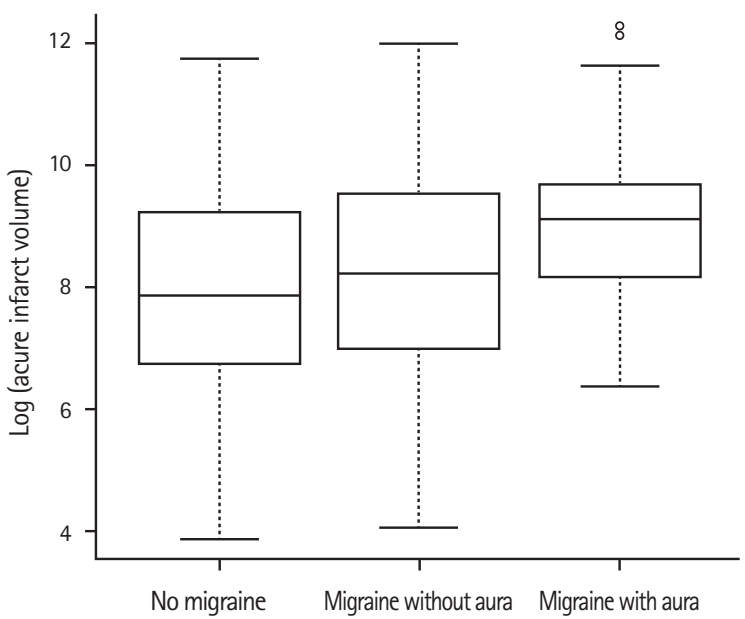

Figure 1. Box-plots of log (acute infarct volume) values by migraine subgroups. In the box-plot the central rectangle spans the first quartile to the third quartile (interquartile range). A bold segment inside the rectangle shows the median and "whiskers" above and below the box show the locations of the minimum and maximum; outside box-plot points are outliers. influence on final infarct volume as covariates, a personal history of migraine was an independent predictor of increased acute infarct volumes $(P<0.001)$. Lesion size, in particular, turned out to be more than once and a half as large in this group compared to the group of patients with no history of migraine ( $M R, 1.80 ; 95 \% \mathrm{Cl}, 1.33$ to 2.45 ). Similarly, the subtype migraine with aura was independently associated with increased acute infarct size in the model including migraine subtypes, lesion volumes being more than 3 and a half times larger in this group in comparison with the reference group of patients with no migraine $(\mathrm{MR}, 3.62 ; 95 \% \mathrm{Cl}, 2.28$ to 5.74$)$. These findings were consistent across sexes (Table 2).

As further support to these findings, we observed an increase of the proportion of patients with any migraine as well as with migraine with aura, and a decrease of the proportion of those with no history of migraine, with increasing quartiles of $\log$ (acute infarct volumes) ( $P$ for trend $<0.001$ ) (Figure 2).

Chronic brain infarcts were present in 75 cases (12.7\%). The volumes of these silent lesions did not differ significantly between patients with migraine (median, $\exp [7.31] / 10^{3=1.5} \mathrm{~cm}^{3}$ [IOR, 0.4 to 4.7]) and patients who did not suffer migraine (median, $\exp [6.91] / 10^{3}=1.0 \mathrm{~cm}^{3}$ [IQR, 0.3 to 3.0]), as well as across migraine subtypes (median, exp [7.74]/10 $=2.3 \mathrm{~cm}^{3}[$ [IQR, 1.4 to $14.9]$ in patients with migraine with aura vs. $\exp [6.81] / 10^{3}=0.9$ $\mathrm{cm}^{3}$ [IQR, 0.04 to 5.0$]$ in patients with migraine without aura).

Table 2. MRs estimates of log (acute infarct volumes) values by linear regression

\begin{tabular}{|c|c|c|c|c|}
\hline \multirow{2}{*}{ Characteristic } & \multicolumn{2}{|c|}{ MR } & \multicolumn{2}{|c|}{ Adjusted MR } \\
\hline & $95 \% \mathrm{Cl}$ & $P$ & $95 \% \mathrm{Cl}$ & $P$ \\
\hline \multicolumn{5}{|l|}{ Entire cohort } \\
\hline Any migraine vs. no migraine & $1.74(1.30-2.35)$ & $2.7 \times 10^{-4}$ & $1.80(1.33-2.45)^{*}$ & $\leq 0.001$ \\
\hline Migraine without aura vs. no migraine & $1.28(0.90-1.82)$ & 0.162 & $1.29(0.91-1.82)^{*}$ & 0.157 \\
\hline Migraine with aura vs. no migraine & $3.04(1.94-4.76)$ & $1.7 \times 10^{-6}$ & $3.62(2.28-5.74)^{*}$ & $7.3 \times 10^{-8}$ \\
\hline \multicolumn{5}{|l|}{ Male } \\
\hline Any migraine vs. no migraine & $1.77(1.16-2.72)$ & 0.009 & $1.95(1.24-3.06)^{*}$ & 0.003 \\
\hline Migraine without aura vs. no migraine & $1.41(0.84-2.35)$ & 0.193 & $1.40(0.83-2.35)^{*}$ & 0.205 \\
\hline Migraine with aura vs. no migraine & $2.66(1.38-5.14)$ & 0.003 & $3.76(1.89-7.49)^{*}$ & $\leq 0.001$ \\
\hline \multicolumn{5}{|l|}{ Female } \\
\hline Any migraine vs. no migraine & $1.59(1.04-2.42)$ & 0.033 & $1.64(1.07-2.52)^{+}$ & 0.024 \\
\hline Migraine without aura vs. no migraine & $1.10(0.68-1.79)$ & 0.696 & $1.14(0.70-1.84)^{+}$ & 0.598 \\
\hline Migraine with aura vs. no migraine & $3.11(1.67-5.78)$ & $4.2 \times 10^{-4}$ & $3.52(1.87-6.62)^{+}$ & $\leq 0.001$ \\
\hline
\end{tabular}

$\mathrm{MR}$, median ratio; $\mathrm{Cl}$, confidence interval.

*Adjusted by age, alcohol consumption, time from stroke onset to magnetic resonance imaging, presumed etiology of brain ischemia (categorized according to the Trial of Org 10172 in Acute Stroke Treatment [TOAST] criteria), patent foramen ovale, large vessel occlusion (occlusion vs. non-occlusion), acute reperfusion therapy (intravenous tissue plasminogen activator and/or mechanical thrombectomy vs. no reperfusion therapy), and arterial recanalization (complete recanalization [Thrombolysis in Cerebral Infarction score of Ilb or higher at the end of the endovascular procedure ${ }^{18}$ ] vs. other); ${ }^{+}$Adjusted as in males $\left({ }^{*}\right)$ and oral contraceptive use. 


\section{Discussion}

In a large series of young patients with ischemic stroke, we have shown that people suffering migraine, especially the subtype migraine with aura, are at risk of developing larger acute brain infarcts, though the burden of silent ischemic lesions does not differ in migraineurs compared with non-migraineurs, nor across migraine subtypes. The data presented here provide evidence that reinforces the experimental observation of a more rapid expansion of the acute ischemic core and, eventually, the development of larger infarcts in transgenic mouse models expressing familial hemiplegic migraine type 1 (FHM1) mutations ${ }^{9}$ and extend the results of previous preliminary works, including ours, ${ }^{11,12}$ linking individual migraine status with increase vulnerability to ischemic brain injury in humans.

The association between migraine and stroke has been subject of considerable debate. Between 2005 and 2018, four meta-analyses included studies involving any stroke and ischemic stroke as outcomes. ${ }^{2,19-21}$ In two of them, the overall risk of stroke was higher in migraineurs. ${ }^{2,19}$ Additionally, three consecutive meta-analyses showed that migraine with aura is consistently associated with a more than 2-fold increased risk of ischemic stroke. ${ }^{2,20,21}$ Such a risk was also around 3-fold higher among women younger than 45 years, ${ }^{2,20} 9$-fold higher among current smokers, ${ }^{2}$ and 7-fold higher among women taking oral contraceptives. ${ }^{2,20}$ More recently, data from Atherosclerosis Risk in Communities (ARIC) study suggested increased incidence of atrial fibrillation and cardioembolic stroke in older migraineurs with visual aura, ${ }_{1}^{22}$ especially among subjects with late onset migraine with aura. ${ }^{23}$ Taken together, despite some conflicting evidence ${ }_{1}^{24-26}$ these findings and our own results support the prevailing idea that migraine is a highly prevalent and perhaps modifiable predisposing factor for brain ischemia, though the exact mechanisms underlying this relationship remain to be clarified. Several biologic pathways implicated in cerebral blood vessel dysfunction have been advocated, including hypercoagulable states, inflammation, platelet and white blood cell endothelial interactions, among the others. ${ }^{27}$ It might be possible that these mechanisms play also a role in the relation between migraine and infarct growth in combination with an underlying condition of increased susceptibility to brain ischemia of migraineurs, thus lending indirect support to the hypothesis of a common pathophysiology for the two diseases, at least in a subset of patients.

Whether migraine may result in increased acute infarct volume outside of the experimental settings has not been properly investigated so far. Actually, only two previous analyses have attempted to provide information on the association between migraine and lesion volume from acute cerebral ischemia in humans. The first is a retrospective analysis of the Norwegian Stroke Research (NORSTROKE) registry, ${ }_{1}^{28}$ which suggested that, as opposed to what observed in our study, migraine may be associated with smaller volume of acute infarcts. The second is a single-center North American study conducted on a relatively small group of acute ischemic stroke patients showing, as suggested from the present analysis, an increased lesion volume among migraineurs with aura. ${ }^{29}$ The reason for these conflicting results may be due, at least to some extent, to methodological issues. As admitted by the authors themselves, the results of the first analysis might be biased by the lack of a rigorous imaging-based volumetric measurement of the brain lesions as well as by the huge number of patients excluded be-
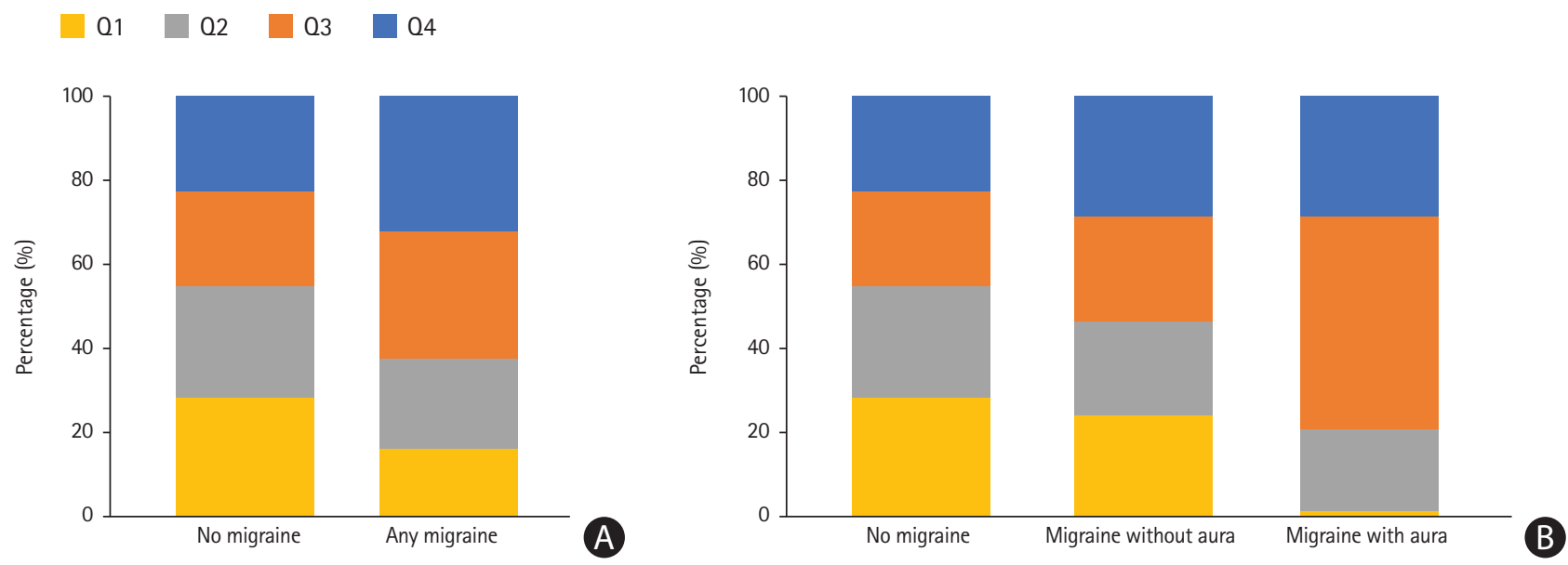

Figure 2. Histogram of percentage of subjects in each quartile of acute infarct volume according to migraine status: (A) any migraine vs no migraine; (B) migraine without aura vs. migraine with aura vs. no migraine. 
cause of more severe stroke symptoms, likely reflecting large infarct size. Also, because of their demographic characteristics, participants to the NORSTROKE registry are probably not the ideal population to investigate. Similarly, the lack of relevant information on clinical and imaging features of the study group, an unavoidable consequence of its characteristics of preliminary report, represents the major shortcoming of the second study and limits the interpretation and generalizability of its findings. Contrary to these previous reports, our study was carried out to test the specific a priori hypothesis on a large number of well-characterized young patients with acute ischemic stroke, migraine status was assessed through direct interview with physicians according to internationally acknowledged criteria, and the same MRI protocol for infarct volume measurement was applied to all study participants. Further strengths of our study include its prospective nature, the central analysis of brain images, and the high interobserver agreement on the measurement of lesion volume.

On the other hand, there are some potential limitations of our analysis worthy of consideration. First, since we did not assess migraine frequency and severity or the frequency of auras, we cannot evaluate whether the observed association differs according to specific migraine patterns. However, whether the clinical features of migraine have any influence on the individual risk of stroke has never been demonstrated. Also, we did not have information on migraine-specific prophylactic drug use, such as, for example, the use of topiramate or lamotrigine, which has been suggested in previous experimental models to protect migraineurs against ischemic injury. ${ }^{30}$ Second, we assessed migraine history by self-report, which raises the possibility of recall bias, creating the potential for misclassification of exposure. This is, however, an unavoidable feature of any epidemiologic study on the relation between migraine and stroke. Third, we cannot exclude that infarct size might depend on other, undetected or hitherto unknown, factors of which migraine is only a marker or an epiphenomenon. Even in this case, however, the hypothesis of a relation between migraine and infarct volume would still be valid and support the external validity of our results. Finally, since participants in this study were aged 18 to 45 years and white, generalizability to other populations might be limited.

\section{Conclusions}

With this caveat in mind, we conclude that the present results support the experimental observations in mice and the sparse data in humans that a personal history of migraine is associated to larger acute infarct volumes, probably through a condi- tion of migraine-associated cerebral hyperexcitability, point toward the possibility that this may be one of the biologic mechanisms underlying the migraine-stroke association, and indirectly strengthen the testable hypothesis that treatment aimed at inhibiting cerebral spreading depolarization might protect migraineurs at risk of stroke.

\section{Supplementary materials}

Supplementary materials related to this article can be found online at https://doi.org/10.5853/jos.2019.00332.

\section{Disclosure}

The authors have no financial conflicts of interest.

\section{Acknowledgments}

The Italian Project on Stroke in Young Adults (IPSYS) is supported by a grant from the Associazione per la Lotta alla Trombosi e alle Malattie Cardiovascolari (ALT). The sponsor had no role in the design and conduct of the study; the collection, management, analysis, and interpretation of the data; the preparation, review or approval of the manuscript; or the decision to submit the manuscript for publication.

\section{References}

1. Jensen $R$, Stovner $\sqcup$. Epidemiology and comorbidity of headache. Lancet Neurol 2008;7:354-361.

2. Schürks M, Rist PM, Bigal ME, Buring JE, Lipton RB, Kurth T. Migraine and cardiovascular disease: systematic review and meta-analysis. BMJ 2009;339:b3914.

3. Tietjen GE. Migraine as a systemic vasculopathy. Cephalalgia 2009;29:987-996.

4. Lee ST, Chu K, Jung KH, Kim DH, Kim EH, Choe VN, et al. Decreased number and function of endothelial progenitor cells in patients with migraine. Neurology 2008;70:1510-1517.

5. Jesurum JT, Fuller CJ, Murinova N, Truva CM, Lucas SM. Aspirin's effect on platelet inhibition in migraineurs. Headache 2012;52:1207-1218.

6. Vanmolkot FH, Van Bortel LM, de Hoon JN. Altered arterial function in migraine of recent onset. Neurology 2007;68: 1563-1570.

7. Bigal $M E$, Kurth $T$, Hu H, Santanello N, Lipton RB. Migraine and cardiovascular disease: possible mechanisms of interaction. Neurology 2009;72:1864-1871.

8. Schwerzmann M, Nedeltchev $K$, Lagger $F$, Mattle HP, Windeck- 
er S, Meier B, et al. Prevalence and size of directly detected patent foramen ovale in migraine with aura. Neurology 2005; 65:1415-1418.

9. Eikermann-Haerter K, Lee JH, Yuzawa I, Liu CH, Zhou Z, Shin $H K$, et al. Migraine mutations increase stroke vulnerability by facilitating ischemic depolarizations. Circulation 2012;125: 335-345.

10. Eikermann-Haerter K. Spreading depolarization may link migraine and stroke. Headache 2014;54:1146-1157.

11. Mawet J, Eikermann-Haerter K, Park KY, Helenius J, Daneshmand $A$, Pearlman $L$, et al. Sensitivity to acute cerebral ischemic injury in migraineurs: a retrospective case-control study. Neurology 2015;85:1945-1949.

12. Pezzini $A$, Busto $G$, Zedde $M$, Gamba M, Zini $A$, Poli $L$, et al. Vulnerability to infarction during cerebral ischemia in $\mathrm{mi}-$ graine sufferers. Stroke 2018;49:573-578.

13. Pezzini A, Grassi M, Lodigiani C, Patella R, Gandolfo C, Casoni $F$, et al. Predictors of migraine subtypes in young adults with ischemic stroke: the Italian Project on Stroke in Young Adults. Stroke 2011;42:17-21.

14. Aho K, Harmsen $P$, Hatano $S$, Marquardsen J, Smirnov VE, Strasser T. Cerebrovascular disease in the community: results of a WHO collaborative study. Bull World Health Organ 1980;58:113-130.

15. Johnson CJ, Kittner SJ, McCarter RJ, Sloan MA, Stern BJ, Buchholz $\mathrm{D}$, et al. Interrater reliability of an etiologic classification of ischemic stroke. Stroke 1995;26:46-51.

16. Pezzini A, Grassi M, Lodigiani C, Patella R, Gandolfo C, Zini A, et al. Predictors of long-term recurrent vascular events after ischemic stroke at young age: the Italian Project on Stroke in Young Adults. Circulation 2014;129:1668-1676.

17. Headache Classification Subcommittee of the International Headache Society. The international classification of headache disorders. Cephalalgia 2004;24(Suppl 1):24-36.

18. Higashida RT, Furlan AJ, Roberts H, Tomsick T, Connors B, Barr J, et al. Trial design and reporting standards for intraarterial cerebral thrombolysis for acute ischemic stroke. Stroke 2003;34:e109-e137.

19. Mahmoud AN, Mentias A, Elgendy AY, Qazi A, Barakat AF,
Saad $M$, et al. Migraine and the risk of cardiovascular and cerebrovascular events: a meta-analysis of 16 cohort studies including 1152407 subjects. BMJ Open 2018;8:e020498.

20. Etminan M, Takkouche B, Isorna FC, Samii A. Risk of ischaemic stroke in people with migraine: systematic review and meta-analysis of observational studies. BMJ 2005;330:63.

21. Spector JT, Kahn SR, Jones MR, Jayakumar M, Dalal D, Nazarian S. Migraine headache and ischemic stroke risk: an updated meta-analysis. Am J Med 2010;123:612-624.

22. Sen S, Androulakis XM, Duda V, Alonso A, Chen LY, Soliman EZ, et al. Migraine with visual aura is a risk factor for incident atrial fibrillation: a cohort study. Neurology 2018;91:e2202e2210.

23. Androulakis XM, Sen S, Kodumuri N, Zhang T, Grego J, Rosa-

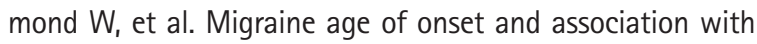
ischemic stroke in late life: 20 years follow-up in ARIC. Headache 2019;59:556-566.

24. Lantz M, Sieurin J, Sjölander A, Waldenlind E, Sjöstrand C, Wirdefeldt K. Migraine and risk of stroke: a national population-based twin study. Brain 2017;140:2653-2662.

25. Kurth T, Gaziano JM, Cook NR, Bubes V, Logroscino G, Diener $\mathrm{HC}$, et al. Migraine and risk of cardiovascular disease in men. Arch Intern Med 2007;167:795-801.

26. Monteith TS, Gardener H, Rundek T, Elkind MS, Sacco RL. Migraine and risk of stroke in older adults: Northern Manhattan Study. Neurology 2015;85:715-721.

27. Mawet J, Kurth T, Ayata C. Migraine and stroke: in search of shared mechanisms. Cephalalgia 2015;35:165-181.

28. Øygarden $H$, Kvistad CE, Bjørk M, Thomassen L, Waje-Andreassen $U$, Naess $H$. Diffusion-weighted lesions in acute ischaemic stroke patients with migraine. Acta Neurol Scand Supp/ 2014;198:41-46.

29. Nahas SJ, Dave HN. Association of history of migraine with aura and larger infarct volume in acute stroke. Cephalalgia 2013;33(8 Suppl):P88.

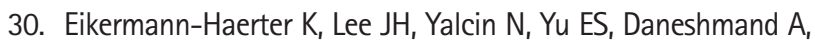
Wei $Y$, et al. Migraine prophylaxis, ischemic depolarizations, and stroke outcomes in mice. Stroke 2015;46:229-236. 


\section{Appendix 1. IPSYS co-investigators (listed by participating centers)}

Department of Clinical and Experimental Sciences, Neurological Clinic, University of Brescia, Brescia, Italy (Alessandro Pezzini, Loris Poli, Valeria De Giuli, Filomena Caria, Alessandro Padovani)

Neurology Unit, Istituti Ospitalieri, Cremona, Italy (Alessia Giossi, Maria Sessa)

Stroke Unit, Vascular Neurology, "Spedali Civili", Brescia, Italy (Massimo Gamba, Mauro Magoni)

Thrombosis Center (Corrado Lodigiani, Paola Ferrazzi, Elena Banfi, Luca Librè) and Emergency Neurology and Stroke Unit (Simona Marcheselli), IRCCS Humanitas Research Hospital, Rozzano-Milano, Italy

Stroke Unit, "Sant'Andrea" Hospital, University of Rome "La Sapienza", Roma, Italy (Alessandra Spalloni, Rosalba Patella, Filomena Di Lisi, Maurizia Rasura)

IRCCS "Neurolesi Bonino-Pulejo" Center, University Hospital, Messina, Italy (Rocco Salvatore Calabrò, Placido Bramanti)

Department of Neurosciences, Psychiatry and Anestesiology, Neurological Clinic, University of Messina, Messina, Italy (Carmela Casella, Paolo La Spina, Francesco Grillo, Masina Cotroneo, Rossella Musolino)

Department of Neurosciences, Rehabilitation, Ophtalmology, Genetics, and Pediatrics, University of Genova, Genova, Italy (Cinzia Finocchi, Maurizio Balestrino, Chiara Bruno, Davide Massucco, Carlo Gandolfo)

Neurology Unit, "Sant'Andrea" Hospital, La Spezia, Italy (Elisa Giorli, Elisabetta Traverso)

Neurology Unit, "Galliera" Hospital, Genova, Italy (Erika Schirinzi, Massimo del Sette)

Neurology Unit, "Ospedale di Circolo," University of Insubria, Varese, Italy (Maria Luisa DeLodovici, Federico Carimati)

Stroke Unit, Neurological Clinic, Nuovo Ospedale Civile "S. Agostino Estense", AUSL, Modena, Italy (Andrea Zini, Anna Maria Simone, Stefania Maffei, Guido Bigliardi, Mauro Gentile, Maria Luisa Dell'Acqua, Francesca Rosafio, Roberta Pentore, Paolo Frigio Nichelli)

Stroke Center, Department of Neurology, "Sacro Cuore Negrar", Verona, Italy (Alessandro Adami)

Neurology Unit, University Hospital "Borgo Trento", Verona, Italy (Monica Carletti, Giampaolo Tomelleri, Paolo Bovi)

Department of Neurosciences, Stroke Unit, University of Torino, Torino, Italy (Paolo Cerrato)

Laboratory of Molecular and Nutritional Epidemiology, Department of Epidemiology and Prevention, IRCCS Mediterranean Neurological Institute, NEUROMED, Pozzilli, Italy (Licia lacoviello, Augusto Di Castelnuovo, Giovanni de Gaetano)

Department of Behavioural and Neurological Sciences, Medical Statistics and Genomics, University of Pavia, Pavia, Italy (Mario Grassi)

Neurology Unit, University Hospital "San Giovanni di Dio e Ruggi d'Aragona", Salerno, Italy (Antonella Toriello, Giampiero Locatelli)

Stroke Unit, Division of Cardiovascular Medicine, University of Perugia, Perugia, Italy (Maurizio Paciaroni, Valeria Caso, Cataldo D'Amore, Giancarlo Agnelli) 
Neurology Unit, "Valduce" Hospital, Como, Italy (Nicoletta Checcarelli, Mario Guidotti)

Neurology Unit (Lucia Tancredi) and Radiology Unit (Laura Demelas, Davide Ferrario), ASST Lariana, Como, Italy

Stroke Unit, Neurology Unit, IRCCS "S. Raffaele" Hospital, Milano, Italy (Giacomo Giacalone, Elisa Zanoli)

Stroke Unit (Anna Cavallini, Alessandra Persico, Andrea Morotti) and Emergency Neurology (Giuseppe Micieli), IRCCS "C. Mondino" Institute, Pavia, Italy

Neurology Unit, University Hospital, Pisa, Italy (Alberto Chiti, Giovanni Orlandi)

Stroke Unit, "G. Brotzu" Hospital, Cagliari, Italy (Valeria Piras, Piernicola Marchi, Maurizio Melis)

Stroke Unit, Department of Neurosciences, ASST Mantova, Italy (Giorgio Silvestrelli, Alessia Lanari, Alfonso Ciccone)

Stroke Unit, Neurology Unit, "S. Chiara" Hospital, Trento, Italy (Valeria Bignamini, Laura Cucurachi)

Neurology Unit, "Cà Foncello" Hospital, ULSS 9, Treviso, Italy (Simone Tonello, Marco Domenico Bonifati)

Neurology Unit, IRCCS "Arcispedale Santa Maria Nuova", Reggio Emilia, Italy (Marialuisa Zedde, Giovanni Malferrari, Norina Marcello)

Neurology Unit, AUSL Romagna, Ravenna, Italy (Enrico Maria Lotti, Pietro Querzani)

Stroke and Neurology Unit, Department of Neuroscience and Rehabilitation, University Hospital, Ferrara, Italy (Cristiano Azzini, Alessandro De Vito, llaria Casetta)

Neurology Unit, "Poliambulanza" Institute, Brescia, Italy (Elisabetta Del Zotto, Paolo Costa, Eugenio Magni)

Neurology Unit, Stroke Unit, ASST Vimercate, Vimercate, Italy (Massimiliano Braga, Sandro Beretta) 
Supplementary Table 1. Relation between infarct volumes, migraine activity, and pain severity

\begin{tabular}{|c|c|c|}
\hline Variable & Median (IQR) & $P$ \\
\hline Migraine & & 1.000 \\
\hline Active migraine & $9.06(1.47-12.14)$ & \\
\hline Inactive migraine & $8.50(3.15-19.45)$ & \\
\hline VAS $^{*}$ & & 0.637 \\
\hline$<7$ & $9.06(1.47-15.89)$ & \\
\hline$\geq 7$ & 8.50 (1.28-17.14) & \\
\hline
\end{tabular}

Data were obtained from a subgroup of 35 patients. Active migraine was defined as at least one migraine attack in the year prior to index stroke; inactive migraine was defined as personal history of migraine but no migraine attacks in the year prior to index stroke.

IQR, interquartile range; VAS, visual analogic scale.

*Self-reported pain intensity, ranging from 0 (no pain) to 10 (maximum pain severity). 